\title{
Caso Clínico. "Systolic anterior motion" (SAM): una causa infrecuente de hipotensión severa en el perioperatorio de cirugía no cardiaca
}

\author{
MARIO CONCHA, VERÓNICA MERTZ K.
}

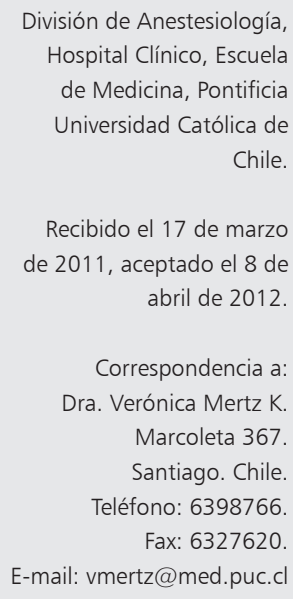

\section{Systolic anterior motion. A report of two cases}

Systolic anterior motion describes the anterior displacement of one or both mitral valve leaflets, obstructing the outflow tract of the left ventricle. It can be a cause of severe hypotension during the intraoperative and postoperative period of non-cardiac surgery. The diagnosis is made with echocardiography. We report two patients with this problem. The first was a 74-year-old male subjected to an incisional hernia repair who presented severe hypotension in the intraoperative period. A transesophageal echocardiography revealed an anterior displacement of the mitral valve anterior leaflet. Epinephrine was discontinued and Norepinephrine and a volume expander were administered, with good response. The second patient was a 64-year-old male undergoing a right liver lobectomy. In the postoperative period, he suffered severe hypotension. A transesophageal echocardiography revealed an anterior displacement of the mitral valve anterior leaflet. Dobutamine was discontinued, volume was administered, and a Norepinephrine infusion was started with good response.

(Rev Med Chile 2012; 140: 1046-1049).

Key words: Hypotension; Mitral valve; Postoperative period.
S ystolic anterior motion (SAM) de la válvula mitral, es una rara causa de deterioro hemodinámico en el período perioperatorio de cirugía no cardiaca. Descrita inicialmente como una característica de la miocardiopatía hipertrófica, puede presentarse en numerosas otras situaciones $^{1-6}$. A pesar de esto, el diagnóstico raras veces se sospecha, y se confirma sólo cuando se realiza una ecocardiografía en un paciente hipotenso que no responde a tratamiento convencional. Reportamos dos pacientes en los cuales una hipotensión severa sólo pudo revertirse cuando se realizó el diagnóstico y las medidas terapéuticas apropiadas establecidas.

\section{Paciente 1}

Paciente de 74 años programado para una herniorrafia incisional supra umbilical. Como único antecedente presentaba una hipertensión arterial no tratada con cifras tensionales de 160/90 $\mathrm{mm} \mathrm{Hg}$, con evidencias electrocardiográficas de hipertrofia ventricular izquierda. Se administró anestesia general, sin incidentes hasta aproximadamente 20 min de cirugía, en que presentó progresiva disminución de la presión arterial hasta $50 / 20 \mathrm{~mm} \mathrm{Hg}$, sin respuesta a la administración de $1.000 \mathrm{ml}$ de ringer lactato y de repetidas dosis de 8-12 mg de efedrina. Se detectó eritema papular difuso que hizo plantear una reacción anafiláctica, y se administraron $300 \mathrm{mg}$ de hidrocortisona, 0,2 y $0,3 \mathrm{mg}$ de adrenalina endovenosa y luego una infusión de $0,25 \mathrm{ug} / \mathrm{kg} / \mathrm{min}$ sin recuperación de la presión arterial. Se realizó una ecocardiografía transesofágica (ETE) que mostró un ventrículo izquierdo hipertrófico, con una válvula aórtica de aspecto y movilidad normal, y con desplazamiento de la valva anterior de la válvula mitral que 
determinaba obstrucción completa del tracto de salida del ventrículo izquierdo (TSVI) durante la sístole. Se administraron rápidamente $1.000 \mathrm{ml}$ de Voluven ${ }^{\circledR}$, se suspendió la infusión de adrenalina y se inició norepinefrina hasta $2,5 \mathrm{ug} / \mathrm{kg} / \mathrm{min}$, con lo que se obtuvo una rápida recuperación de condiciones hemodinámicas aceptables. Al término de la cirugía, el paciente se trasladó a la unidad de cuidados intensivos, donde se disminuyó gradualmente hasta suspender la noradrenalina y se extubó $6 \mathrm{~h}$ después. Alta al quinto día post operatorio.

\section{Paciente 2}

Paciente de 64 años, hipertenso en tratamiento con atenolol con lo que mantenía cifras tensionales de 140/85 mm Hg. El ecocardiograma mostraba una buena función sistólica e hipertrofia ventricular izquierda. Se realizó una lobectomía hepática derecha, sin incidentes de importancia durante el intraoperatorio. Aproximadamente a las $12 \mathrm{~h}$ del post operatorio presentó disminución de presión arterial hasta $80 / 50 \mathrm{~mm} \mathrm{Hg}$ y frecuencia cardiaca de 135, sin respuesta a la administración de $500 \mathrm{ml}$ de Voluven ${ }^{\circledR}$. Se inició infusión de dobutamina que se aumentó hasta $7 \mathrm{ug} / \mathrm{kg} / \mathrm{min}$, observándose mayor deterioro de las condiciones hemodinámicas, por lo que se colocó un catéter de arteria pulmonar que mostraba una presión de capilar pulmonar (PCP) de $18 \mathrm{mmHg}$, y un índice cardiaco de 1,2 lt/ $\mathrm{m}^{2}$. Se realizó un ETE que mostraba un ventrículo izquierdo hipertrófico, con una cavidad pequeña, con buena función global y sin alteraciones de motilidad segmentaria, y con una válvula aortica de aspecto y movilidad normal. Durante el sístole, la valva anterior de la válvula mitral se desplaza obstruyendo el 70\% del TSVI, y produciendo una insuficiencia mitral (IM) moderada. Se hizo el diagnóstico de SAM, se administraron $1.000 \mathrm{ml}$ de Voluven ${ }^{\circledR}$, se inició infusión de noradrenalina hasta $2 \mathrm{ug} / \mathrm{kg} / \mathrm{min}$, se suspendió la dobutamina, y se administran $3 \mathrm{mg}$ de metoprolol. Dentro de los siguientes 20 min se observa disminución de la obstrucción del TSVI y de la IM, la presión arterial se estabilizó en 100/60 mm Hg y la frecuencia cardiaca en 100. Doce horas después se presentó nuevo episodio de compromiso hemodinámico de características similares. Por la posibilidad de la existencia de un hemoperitoneo que pudiera perpetuar una condición de hipovolemia que favoreciera el desarrollo de un SAM, se realizó una laparotomía exploradora, encontrándose 300 $\mathrm{ml}$ de sangre antigua, sin identificarse un sitio de sangrado. Se aportó volumen, la norepinefrina se aumentó de 0,05 a $1,5 \mathrm{ug} / \mathrm{kg} / \mathrm{min}$, restableciéndose PA de 100/70 mm Hg, PCP de $22 \mathrm{~mm} \mathrm{Hg}$, e IC 3,2 $\mathrm{lt} / \mathrm{m}^{2}$. No se observaron otros problemas hemodinámicos en el post operatorio. El paciente fue dado de alta al día 30 debido a colecciones intraabdominales que requirieron drenaje percutáneo.

\section{Discusión}

SAM está descrito como la obstrucción del TSVI por el desplazamiento anterior de una o ambas valvas de la válvula mitral. Como resultado de esto puede asociarse IM. Ambas características se encontraban presentes en los pacientes reportados. Dado que las imágenes fueron consideradas características, dada la gravedad de la condición clínica y la rápida respuesta a las medidas terapéuticas, no se realizaron otras determinaciones para confirmar el diagnóstico. Sin embargo, una mayor rigurosidad diagnóstica hubiese hecho necesario el uso de Doppler color que evidenciaría una aceleración del flujo en el TSVI previo a la obstrucción y aparición de flujo turbulento post estenótico ${ }^{7}$. El uso de modo $\mathrm{M}$ a nivel de la válvula mitral también hubiese ayudado a precisar el grado de desplazamiento anterior del velo mitral. La falta de respuesta sin embargo, hubiese hecho necesario descartar otras causas como una estenosis aortica subvalvular o una isquemia miocárdica. Estas mediciones sin embargo, pueden ir más allá de lo que un médico no frrcuentemente expuesto a esta técnica pudiera realizar, y enfatizan la importancia de la evaluación cualitativa de las estructuras cardiacas y de su función, lo que en otras situaciones ha mostrado ser adecuado para la evaluación de la función cardiaca ${ }^{8}$. SAM es un proceso dinámico que puede estar determinado por ciertas condiciones anatómicas o patológicas 9 . En otros casos, modificaciones de la precarga, post carga o contractilidad, pueden modificar la arquitectura ventricular, permitiendo la obstrucción del TSVI ${ }^{5,6}$. Todas estas condiciones se presentan frecuentemente en el período perioperatorio como resultado de efecto de drogas, estimulación simpática, o hipovolemia. Los pacientes reportados presentaban varias de las condiciones 
mencionadas: hipertrofia ventricular izquierda, importante disminución de la precarga, secundaria a vasodilatación severa en uno y a pérdida de volumen intravascular en otro, determinando ambas situaciones disminución del retorno venoso, y disminución de la post carga secundaria a vasodilatación por la reacción anafiláctica en uno, y secundaria al uso de dobutamina en el otro. En ambos el uso de $\beta$ adrenérgicos antes de hacer el diagnóstico probablemente contribuyó a agravar la obstrucción del TSVI. En el segundo paciente, se agrega la posibilidad que la dobutamina, a través de su efecto vasodilatador pudiera haber actuado como un factor agravante adicional.

Considerando lo inespecífico de la signología del cuadro y de la información obtenida con la monitorización habitual, la que puede llevar a establecer medidas terapeúticas absolutamente contrarias a las correctas, el diagnóstico de SAM requiere de un alto índice de sospecha y la imprescindible confirmación ecocardiografica, idealmente ETE. La información obtenida del catéter de arteria pulmonar puede ser difícil de interpretar. El aumento de la PCP puede explicarse por disminución de la distensibilidad secundario a la hipertrofia ventricular, y la disminución del IC por la obstrucción del TSVI, y no por disfunción ventricular. En ausencia de la información obtenida con el ETE, frente a mediciones hemodinámicas sugerentes de claudicación ventricular izquierda, la disminución del aporte de volumen y la administración de un inótropo con efecto vasodilatador parece una muy buena indicación. Desafortunadamente, en un SAM, estas medidas terapéuticas son exactamente las opuestas a las requeridas. Este hecho enfatiza la importancia de la ETE, que permite hacer el diagnóstico y establecer el tratamiento correcto. El conocimiento de la existencia de factores que pueden predisponer para la aparición de SAM como: presencia de una miocardiopatía hipertrófica, hipertensión arterial con hipertrofia ventricular, o algunas situaciones más infrecuentes como pacientes diabéticos sometidos a estimulación $\beta$ adrenérgica, o incluso en pacientes con infarto miocárdico ${ }^{9}$, deben hacer plantear la posibilidad de este diagnóstico como la posible causa de un cuadro de un severo compromiso hemodinámico, especialmente cuando no se dispone con facilidad de ecocardiografía, y en forma empírica se plantea el uso de vasoconstrictores. En situaciones en las cuales se considera el uso de $\beta$ bloqueadores, la posibilidad de eliminar un mecanismo compensatorio, que puede ser de gran importancia cuando se trata de otro cuadro, hace mandatorio la confirmación del diagnóstico.

La ETE ha sido recomendada para tratar de determinar la causa de un compromiso hemodinámico grave durante cirugía no cardiaca ${ }^{10}$.

Se reportan dos casos de SAM en los cuales el diagnóstico sólo pudo ser realizado utilizando ETE, lo cual permitió modificar el tratamiento en curso y establecer las medidas adecuadas. Cuadros como SAM, que no pueden ser diagnosticados sin ecocardiografía, en los cuales la información obtenida con los medios de monitorización habituales e incluso un catéter de arteria pulmonar, pueden hacer parecer indicado terapias que pueden agravar la situación, nos obliga a tomar conciencia de la necesidad de incorporar la ETE como un medio de diagnóstico habitual en el período perioperatorio de cirugía no cardiaca. Finalmente, se debe insistir en la importancia del apoyo cardiológico para optimizar los beneficios de la ETE en manos de intensivistas o anestesiólogos, tanto para el entrenamiento como para la correcta interpretación de las imágenes.

\section{Referencias}

1. Doi YL, McKenna WJ, Oakley CM, Goodwin JF. 'Pseudo' systolic anterior motion in patients with hypertensive heart disease. Eur Heart J 1983; 4: 838-45.

2. Cueto L, Arriaga J, Zinser J. Echocardiographic changes in pheochromocytoma. Chest 1979; 76: 600-1.

3. Henein MY, O'Sullivan C, Sutton GC, Gibson DG, Coats AJ. Stress-induced left ventricular outflow tract obstruction: a potential cause of dyspnea in the elderly. J Am Coll Cardiol 1997; 30: 1301-7.

4. Maraud L, Gin H, Roudaut R, Aubertin J, Bricaud H. Echocardiographic study of left ventricular function in type 1 diabetes mellitus: hypersensitivity of betaadrenergic stimulation. Diabetes Res Clin Pract 1991; 11: 161-8.

5. Aniskevich S, Shine TS, Feinglass NG, Stapelfeldt WH. Dynamic left ventricular outflow tract obstruction during liver transplantation: the role of transesophageal echocardiography. J Cardiothorac Vasc Anesth 2007; 21: 577-80.

6. Luckner G, Margreiter J, Jochberger S, Mayr V, Luger T, Voelckel W, et al. Systolic anterior motion of the mitral valve with left ventricular outflow tract obstruction: 
three cases of acute perioperative hypotension in noncardiac surgery. Anesth Analg 2005; 100: 1594-8.

7. Savage R, Correia, R. In: Konstant S, Shernan, S, Oka, Y, ed. Clinical Tranesophageal Echocardiography A ProblemOriented Approach. Philadelphia: Lippincott Williams \&Wilkins, 2003: 379-99.

8. Melamed R, Sprenkle MD, Ulstad VK, Herzog CA, Leatherman JW. Assessment of left ventricular function by intensivists using hand-held echocardiography. Chest 2009; 135: 1416-20.

9. Luckie M, Khattar RS. Systolic anterior motion of the mitral valve-beyond hypertrophic cardiomyopathy. Heart 2008; 94: 1383-5.

10. Fleisher LA, Beckman JA, Brown KA, Calkins H, Chaikof
EL, Fleischmann KE, et al. ACC/AHA 2007 Guidelines on Perioperative Cardiovascular Evaluation and Care for Noncardiac Surgery: Executive Summary: A Report of the American College of Cardiology/American Heart Association Task Force on Practice Guidelines (Writing Committee to Revise the 2002 Guidelines on Perioperative Cardiovascular Evaluation for Noncardiac Surgery) Developed in Collaboration With the American Society of Echocardiography, American Society of Nuclear Cardiology, Heart Rhythm Society, Society of Cardiovascular Anesthesiologists, Society for Cardiovascular Angiography and Interventions, Society for Vascular Medicine and Biology, and Society for Vascular Surgery. J Am Coll Cardiol 2007; 50: 1707-32. 